\title{
Erratum: Sudden stress relaxation in compound semiconductor thin films triggered by secondary phase segregation [Phys. Rev. B 92, 155310 (2015)]
}

R. Mainz, H. Rodriguez-Alvarez, M. Klaus, D. Thomas, J. Lauche, A. Weber, M. D. Heinemann, S. Brunken, D. Greiner,

C. A. Kaufmann, T. Unold, H.-W. Schock, and C. Genzel

(Received 14 March 2016; published 6 April 2016)

DOI: 10.1103/PhysRevB.93.159902

We noticed that an incorrect version of the equation for the relaxed lattice spacing $d_{\mathrm{r}}$ was included in the Supplemental Material (Sec. 3). The original, incorrect, equation was as follows:

$$
d_{\mathrm{r}}=d_{\Psi} \frac{\left(\frac{1}{v}+1\right)}{2 \sin ^{2} \Psi}+d_{0^{\circ}}\left(1-\frac{\left(\frac{1}{v}+1\right)}{2 \sin ^{2} \Psi}\right) .
$$

The correct equation is

$$
d_{\mathrm{r}}=d_{\Psi} \frac{2}{\left(\frac{1}{v}+1\right) \sin ^{2} \Psi}+d_{0^{\circ}}\left(1-\frac{2}{\left(\frac{1}{v}+1\right) \sin ^{2} \Psi}\right) .
$$

The Supplemental Material has been amended accordingly. This mistake, however, does not affect the evaluations and conclusions in the main paper, since for all calculations of $d_{\mathrm{r}}$ [depicted in Figs. 2(a) and S5] the correct equation was used.

We are grateful to Helena Stange for pointing this out. 